Running head: EFFECTS OF INSTRUCTIONS

\author{
The Effects of Instructions on Mothers' Ratings of \\ Child Attention-Deficit/Hyperactivity Disorder Symptoms \\ Johnston, C., Weiss, M., Murray, C., \& Miller, N.
}

AUTHOR PREPUBLICATION DRAFT 


\begin{abstract}
We examined whether instructional materials describing how to rate child ADHD symptoms would improve the accuracy of mothers' ratings of ADHD symptoms presented in standard child behavior stimuli, and whether instructions would be equally effective across a range of maternal depressive symptoms and family incomes. A community sample of 100 hundred mothers with 5 to 12 year old sons were randomly assigned to either receive or not receive the instructions. All mothers watched standard DVDs of boys displaying nonproblem behavior, ADHD symptoms, ADHD plus oppositional behaviors, or ADHD plus anxious behaviors, and then rated the ADHD symptoms of the boys in the DVDs. These ratings were compared to ratings of the boys' ADHD symptoms made by objective coders. Results indicated an interaction such that the instructional materials improved the agreement between mothers' and coders' ratings, but only for mothers at lower family income levels. The instructional materials improved all mothers' open-ended responses regarding knowledge of ADHD. All mothers rated more ADHD symptoms in boys with comorbid oppositional or anxious behaviors, and this effect was not reduced by the instructional materials. The potential utility of these instructions to improve the accuracy of ratings of child ADHD symptoms is explored.
\end{abstract}

Keywords: Attention-Deficit/Hyperactivity Disorder, ADHD, parent ratings, assessment 


\section{The Effects of Instructions on Mothers' Ratings of Child Attention-Deficit/Hyperactivity Disorder Symptoms}

Attention-Deficit/Hyperactivity Disorder (ADHD) is a serious child mental health concern characterized by developmentally-inappropriate levels of inattention and hyperactivity/impulsivity. With a prevalence of 3 to $7 \%$ of elementary-school aged children, predominantly males (American Psychiatric Association (APA), 2000), it is associated with significant concurrent and long-term impairment (Barkley, 2006). As is the case with many childhood disorders, parental reports of the child's behavior play a crucial role in ADHD assessment. Within a complete, multi-method and multi-source assessment of ADHD, parental reports of the child's behavior on checklists that ask about symptoms of ADHD are considered a central component (Pelham, Fabiano, \& Massetti, 2005). The instructions provided with such rating scales are minimal, and leave considerable room for parents to misinterpret or to construe items in a manner that is different from the meaning intended by the clinician or researcher. This study examines whether instructions describing ADHD and how to rate its symptoms can increase the accuracy of parents' assessments of child ADHD symptoms.

Parents have invaluable knowledge about their child's behavior and their role in the assessment of ADHD cannot be under-estimated. However, parental reports of a child's ADHD symptoms often do not agree well with the reports of others such as teachers, clinicians, objective observers, or the child (Collett, Ohan, \& Myers, 2003; Power et al., 1998; Wolraich et al., 2004). Indeed, studies have suggested that rater source variance (i.e., whether ratings are done by parents or teachers) can be as influential as trait source variance (i.e., differences due to the children being rated) on rating scales assessing ADHD symptoms (Gadow et al., 2004;

Gomez, Burns, Walsh, \& De Moura, 2003). Thus, clinicians and researchers assessing childhood 
ADHD are faced with a situation where parental reports are essential to the process, but are often discrepant from other assessment information.

Several factors may account for the lack of agreement between parents' reports of child ADHD and information provided by other means. Certainly, parents, teachers, and clinicians have experience with children in different contexts, and to the extent that the child's behavior varies across these different settings, the ratings will reflect this difference. However, agreement between mothers' and fathers' ratings of childhood ADHD, even in the same home context remains at only a moderate level (e.g., intraclass correlation coefficients of approximately .40; Langberg et al., 2010). Even more striking, Solanto and Alvir (2009) recently reported relatively low levels of intra-rater reliability for items even with the same reporter completing the same items on two ADHD scales administered within the same questionnaire package. We suggest that characteristics of the rating scales themselves, in particular potential ambiguity in the meaning of items, may be contributing to this lack of agreement across and within raters. In the present study, we explore the possibility that the low reliability between a parent's report of child ADHD symptoms and other criteria (e.g., teacher reports, observations) reflects the difficulties parents encounter in determining the meaning of items on ADHD rating scales.

The most widely used ADHD rating scales include the ADHD-IV Rating Scale (DuPaul, Power, Anastopoulos, \& Reid, 1998), the Disruptive Behavior Rating Scale (Pelham, Gnagy, Greenslade, \& Milich, 1992), the SNAP (Bussing et al., 2008), the Child Symptom Inventory - 4 (Gadow \& Sprafkin, 2002), and the Conners Rating Scales (Conners, Sitarenios, Parker, \& Epstein, 1998). Each of these rating scales lists the symptoms of ADHD as found in the Diagnostic and Statistical Manual - IV (APA, 2000) and asks parents to rate the extent to which their child displays these symptoms. The items include only brief DSM descriptions of the 
symptoms (e.g., "often has difficulty organizing tasks or activities"), with little or no elaboration. When parents complete these scales, they may interpret the symptoms in a manner that is different from that intended (e.g., Burns, Walsh, Gomez, \& de Moura, 2003). Parents may misinterpret the item "often does not seem to listen when spoken to directly" as referring to noncompliant behaviors or parents may rate the item "fails to pay attention" as not present, because the child spends many hours on activities, such as videogames. Or, parents may rate the item "often loses things necessary for tasks or activities" as never occurring because "he never really loses things, we always find them somewhere." To the extent that parents differ from each other, or from teachers, observers, and clinicians in their interpretations of these items, the agreement between sources and the accuracy of parents' ratings will be lowered. We argue that the descriptions of the core symptoms in parent rating scales may be misinterpreted by parents, and this may lead to either over or under diagnosis.

This study reports on the development of instructional materials designed to assist parents in interpreting the symptom descriptions on ADHD rating scales in a manner consistent with intended DSM meanings, and an evaluation of whether these instructional materials improve the agreement between reports of child behavior made by mothers and objective observers. We predicted that mothers who received the instructional materials would provide ratings of standardized child behavior stimuli that were more closely aligned with the ratings provided by objective coders than mothers who did not receive the instructional materials. We also examined whether the instructions would improve the accuracy of mothers' open-ended descriptions of ADHD. In studying the influence of instructions on mothers' ratings of child ADHD symptoms, we wished to hold constant the child behavior being rated. Therefore, we generated standard child behavior stimuli that were viewed and rated by the mothers in the study. As a first step in 
evaluating these instructional materials, we chose to test them in a sample of mothers recruited from the general community. In contrast to parents whose children have already been diagnosed with ADHD, mothers in the community are assumed to be relatively unfamiliar with ADHD. Thus, we judged this group as representative of parents in the initial stages of seeking help for their children and an appropriate sample for preliminary testing of the instructional materials for use in the initial phases of assessment of ADHD. At this initial stage in testing the instructional materials, we focused on mothers of sons given the relatively higher likelihood of boys being referred and assessed for ADHD compared to girls (APA, 2004).

Instructional materials may be more important for some parents than for others. Specifically, variables such as socioeconomic and mental health status may interact with the influence of the instructional materials, making the instructions of greatest benefit to parents who are more socially disadvantaged or depressed. Studies support a link between socioeconomic status and parental reports of child ADHD symptoms and treatment outcomes (e.g., Larson, Russ, Kahn, \& Halfon, 2011; Rieppi et al., 2002). Socioeconomic level may impact a variety of factors related to parents' identification of child ADHD symptoms, including knowledge of ADHD (Bussing, Gary, Mills, \& Wilson Garvan, 2007) or access to mental health information and treatments (Larson et al., 2011). Other studies consistently relate maternal depressive symptoms to inaccuracies or biases in reports of child ADHD symptoms (Chi \& Hinshaw, 2002; Johnston \& Short, 1993). Therefore, we hypothesized that instructions describing ADHD and how to rate the symptoms in children would be of greater benefit to mothers of lower socioeconomic status or with higher levels of depressive symptoms as these mothers might be those most in need of health information or most likely to show existing biases in their ratings. 
The presence of co-occurring problems in the child also influences parents' ratings of ADHD behaviors. Several studies have shown that ratings of ADHD symptoms are inflated for children who exhibit both ADHD and oppositional defiant behaviors (e.g., Abikoff, Courtney, Pelham, \& Koplewicz, 1993; Freeman, Johnston, \& Barth, 1997; Hartung, Van Pelt, Armendariz, \& Knight, 2006; Hartung et al., 2010; Jackson \& King, 2004). However, the influence of other comorbid behaviors has not been examined. At least a third of children with ADHD also have an internalizing disorder, such as anxiety or depression (e.g., Angold, Costello, \& Erkanli, 1999; Bauermeister et al., 2007). Whether the presence of child internalizing problems exerts an influence on parent's ratings of the child's ADHD behaviors is unknown. It is possible that some child anxiety symptoms are mistaken for characteristics of ADHD (e.g., difficulty making decisions being interpreted as inattention; agitation being misinterpreted as hyperactivity), with the result that children with both ADHD and anxious behaviors are reported as having higher levels of ADHD symptoms than children showing only ADHD behaviors. Alternately, in a child with both ADHD and anxious behaviors, some ADHD behaviors may be misinterpreted as anxiety (e.g., failing to finish a task due to anxiety), and the parent may consequently report lower levels of ADHD in such children. Thus, we predicted that co-occurring oppositional behaviors would increase mothers' ratings of child ADHD symptoms, but left open the question of whether co-occurring anxious behaviors would increase or decrease ADHD ratings. Finally, because the instructional materials are designed to clarify how ADHD symptoms differ from symptoms of anxiety or oppositional disorders, we predicted that the instructions would reduce the impact of the co-occurring behaviors on mothers' ratings of ADHD symptoms.

In summary, this study predicted that instructions regarding how to complete ratings of ADHD symptoms would improve the accuracy of mothers' ratings of standard presentations of 
ADHD child behaviors and their knowledge of ADHD. We also predicted interactions of the influence of instructions with maternal socioeconomic status and depressive symptoms, such that larger effects would be found for mothers with lower socioeconomic status or more depressive symptoms. Finally, we predicted that co-occurring oppositional, and perhaps anxious, child behaviors would increase mothers' ratings of child ADHD behaviors, but that these effects would be attenuated in the presence of the instructional materials.

\section{Methods}

\section{Participants}

One-hundred and twenty-three mothers of 5 to 12 year old nonproblem boys were recruited from the community, using notices on internet lists, in community newspapers, and public places such as coffee shops or community centers. This child age range represents the common age for ADHD assessment, and our focus on mothers and sons is based on the gender ratio of ADHD (APA, 2000) and the fact that mothers are more consistently involved in the assessment of childhood problems than are fathers (Lee, 2006). Of the 123 mothers who were assessed as eligible for the study, 19 were excluded ( 7 declined to participate and 12 were not able to be scheduled before the study closed). One-hundred and four mothers were randomized to either receive the instructional materials (Instruction Group, $n=49$ ) or not (No Instruction Group, $n=55$ ). In the No Instruction Group, four mothers were excluded due to missing information (two failed to complete the ratings of child behavior and two did not complete other measures) leaving 51 mothers in the No Instruction Group. Demographic and descriptive characteristics of the two groups of mothers are shown in Table 1. There were no significant differences across the groups in ethnicity, child age, or maternal age.

\section{Instructional Materials}


The instructional materials were created to provide general instructions to aid in ratings. For example, parents are told to rate the symptoms by comparing the child to other children of the same age and sex, to not rate behaviors that occur in limited situations such as only when the child is tired, and to rate the child's behavior as it would be without adult intervention or help. In addition, specific examples of each symptom were provided, along with examples of behaviors that would not be rated as ADHD symptoms (e.g., behaviors due to learning problems or oppositionality). The materials were created to be appropriate for the behavior of elementaryschool age children and to be within the reading and comprehension levels of parents with a grade 8 or higher education. The first three authors, all with extensive clinical experience with ADHD, began by generating definitions and examples of ADHD symptoms and examples of behaviors that do not meet symptom definitions. Subsequently, we surveyed a panel of 18 child psychiatrists and psychologists who were recognized experts in ADHD to ascertain ratings regarding the appropriateness and clarity of these materials. These experts had an average of 21 years of experience in the area of ADHD. These experts rated the instructional materials on 7 point scales $(1=$ not at all; $7=$ very $m u c h)$ assessing whether they were accurate (mean rating 5.79), appropriate for a grade 8 reading level (mean rating 5.41), appropriate for both boys and girls (mean rating 5.94), engaging (mean rating 5.44), and helpful/informative (5.38). The experts also offered occasional suggestions for improvements in wording and these were incorporated as appropriate. We then prepared the instructional materials in the form of a pamphlet and an accompanying computer "slide show" presentation with a voice-over narration ${ }^{1}$. Mothers were randomly assigned to receive or not receive the instructions prior to viewing and

\footnotetext{
${ }^{1}$ Instructional materials are available from first author on request.
} 
rating the child behavior stimuli described in the following section.

\section{Child Behavior Stimuli and Child Behavior Ratings}

Because we wished to determine the influence of the instructional materials on the accuracy of mothers' ratings, variance due to differences in the behavior of the mothers' own children needed to be controlled. Therefore, we employed standard child behavior stimuli that all mothers rated, rather than having each mother rate her own child. We recruited eight boys with ADHD (between 7 and 10 years of age) and video recorded them engaging in a series of semiscripted home situations designed to elicit ADHD symptoms, anxiety and oppositional symptoms, as well as nonproblem behaviors. Boys were shown engaged in various home situations (e.g., homework, putting dishes away). Occasionally the boy's mother or her voice was included in the tape, but other children or adults were not present. The research team edited each boy's video recording and selected short clips (3 to 27 seconds) of specific behaviors that illustrated the described symptoms/behaviors and were clear, audible, and did not include anyone other than the child in the clip. These clips were then viewed by a large group of undergraduate students and rated as to whether or not the behavior intended (e.g., fidgeting) was displayed. These ratings provided a consensus regarding the behaviors that were portrayed. Based on these ratings, for each child we selected the best clips and these were combined to create four DVDs for each of the eight children (for a total of 32 DVDs). The four DVDs contained either a 2 to 1 ratio of ADHD and nonproblem behaviors (ADHD condition), a 2 to 1 ratio of ADHD and oppositional behaviors (ADHD+ODD condition), a 2 to 1 ratio of ADHD and anxious behaviors (ADHD+ANX condition), or all nonproblem behaviors (NONPROB condition). For each child, the quantity and content of ADHD behaviors depicted were identical across all DVDs (except the NONPROB DVDs). 
Each mother watched DVDs from all eight of the children, with two of the DVDs in each of the above categories (ADHD, ADHD+ODD, ADHD+ANX, NONPROB). Which DVD from each boy was shown was counterbalanced across mothers and the order of presentation of the boys' DVDs was randomized across mothers. Following each DVD, the mother rated the child's ADHD symptoms on a slightly modified ${ }^{2}$ version of the ADHD-IV Rating Scale (DuPaul et al., 1998) that asked mothers the extent to which the child on the DVD displayed each ADHD symptom, on a scale of $0=$ Not at all to $3=$ Very much. The ADHD-IV Rating Scale is widely used in ADHD assessment, and demonstrates satisfactory reliability and validity (DuPaul et al., 1998). For each DVD, mothers' ratings were summed across all symptoms to create a total rating score. In addition, to explore whether instructions or comorbid behaviors might differentially influence mothers' ratings of inattentive vs. hyperactive/impulsive symptoms, rating scores also were calculated separately for inattentive and for hyperactive/impulsive symptoms.

To generate an objective criterion describing the ADHD symptoms displayed on each DVD, three trained coders provided ratings of each boy's four DVDs. Coders were trained using a modified version of the Attention Deficit/Hyperactivity Problems scale of the Direct Observation Form (McConaughy \& Achenbach, 2009). Specific ADHD symptoms were rated on a 4-point scale $(0=$ not at all; $3=$ very $m u c h)$ that incorporated information about frequency and intensity. Nine inattentive symptoms (e.g., distracted by external stimuli, loses things) and 11 hyperactive/impulsive symptoms (e.g., fidgets, responds before instructions are completed) were assessed using the coding system. The child stimulus DVDs were coded independently by the three coders, and the intraclass correlation coefficient between the coders for the total rating of

\footnotetext{
${ }^{2}$ Because mothers would be rating only a brief sample of the child's behavior, we used the scale anchors of "not at all, just a little, pretty much, and very much", rather than the anchors provided with the ADHD-IV Rating Scale.
} 
ADHD symptoms was .99 , with .98 and .98 for the number of Inattention and Hyperactive/Impulsive symptoms, respectively.

To assess the mothers' accuracy in rating the DVDs, scores were calculated for each DVD the mother watched by subtracting the objective coder's total symptom score for the DVD from the mother's total rating score for the same DVD (Inaccuracy score). Mother Inaccuracy scores also were calculated for inattentive and hyperactive/impulsive symptoms separately. These scores were averaged across the two DVDs of each type (ADHD, ADHD+ANX, ADHD+ODD, and NONPROB) generating four Inaccuracy scores for each mother, as well as four Inaccuracy scores for inattentive symptoms and four for hyperactive-impulsive symptoms.

\section{Knowledge of ADHD}

Further assessment of the influence of the instructional materials on mothers' knowledge of ADHD was conducted by asking mothers open-ended questions regarding how they would describe ADHD, the problems that children with ADHD have, and the differences they see between children with and without ADHD. These questions were asked immediately after the mothers had completed ratings of the child behavior DVDs. The research assistant wrote down the mother's responses and these were subsequently coded by three coders blind to the group assignment of the mother (and different from the coders of the child DVDs). The frequency of three types of statements in mothers' responses was assessed: 1) Correct Terms and Guidelines used to describe ADHD symptoms and how to rate the symptoms (e.g., use of term attention deficit, reference to needing to rate child according to other children of the same age), 2) General Descriptions of ADHD (e.g., mention of distractibility, child being "on the go"), and 3) Inaccurate Statements regarding the nature or etiology of ADHD (e.g., statements that ADHD behaviors can be explained by bad parenting or is a current fad). Two of the three coders were 
randomly assigned to each mothers' statement and intraclass correlation coefficients indicated good inter-coder reliability for Correct Terms and Guidelines, ICC $=.77$ and General

Descriptions, ICC $=.88$. The reliability of the Inaccurate Statements category was not good, ICC $=.42$, probably reflecting its very low base rate.

\section{Evaluation of the Instructional Materials}

Mothers in the Instruction Group, following their ratings of the child behavior DVDs, rated both the written pamphlet and the slide/audio presentation on 7 -point scales $(1=$ not at all and $7=$ completely) assessing whether the materials were easy to understand, engaging or interesting, and would be helpful or informative to parents of children with ADHD.

\section{Moderators}

Measures were included to test the predictions that socioeconomic status and depressive symptoms would moderate the influence of instructions. Mothers reported their depressive symptoms on the Depression subscale of the Brief Symptom Inventory (BSI; Derogatis, 1993). The BSI Depression subscale has good psychometric properties and, in the current sample, the internal consistency was good, Cronbach's $\alpha=.86$. Family income served as the index of family socioeconomic status. Mothers reported their family income over the past year on a 9-point scale with categories increasing in $\$ 15,000$ increments from less than $\$ 5,000$ to $\$ 200,000$ and higher.

\section{Potential Covariates}

Given that the level of behavior problems in the mothers' own child might influence their perceptions of ADHD symptoms in the children on the DVDs, mothers reported on their own child's behavior problems on the Strengths and Difficulties Questionnaire (SDQ; Goodman, 1997). This brief questionnaire for 3-16 year-old children has good psychometric properties. In this sample, the Cronbach's $\alpha$ was .63 for the Emotional Problems scale, .59 for Conduct 
Problems , .78 for Hyperactivity, .63 for Peer Problems, and for the Total Problem score the alpha was .82. We also assessed the mothers' familiarity with ADHD prior to the study, in order to examine any possible differences across the groups in this knowledge. Mothers rated their level of familiarity with ADHD on a 4-point scale ranging from "not at all" to "completely." They also indicated whether they had gained information regarding ADHD from media, scientific/medical literature, knowing someone with ADHD, or having a family member with ADHD (yes or no questions).

\section{Procedures}

The research was approved by our university's ethical review board. When mothers called the lab, they were provided with a description of the study and, if they were interested in participating, a time was arranged either for them to come to the lab or for a research assistant to visit their home. Sixty-three percent of mothers participated in the lab, and there was no difference in rates of lab vs. home participation across the two groups. Following informed consent, mothers randomly assigned to the Instruction Group read the instructional pamphlet and watched the slide presentation. Then, they proceeded to watch the eight DVDs and complete ratings of ADHD symptoms for each boy. Mothers were free to refer to the instructional pamphlet throughout their participation. Mothers assigned to the No Instruction Group were given a brief description of ADHD, and then proceeded immediately to watching the child DVDs and making their ratings. Following the DVDs, mothers in the Instruction Group rated both the instructional slide show and pamphlet in terms of their ease of understanding, interest-level, and overall helpfulness. Finally, all mothers reported on their own child's behavior.

\section{Analysis Plan}


We first examined group differences on possible covariates and the moderators, using $t$ test comparisons. To address hypotheses regarding the influence of the instructions on mothers' ratings of the child behavior DVDs, and the interactions of instructional group with income and depressive symptoms, the mother's Inaccuracy scores were examined using hierarchical linear modeling, with the behavior ratings for the four types of child behavior (ADHD, ADHD+ANX, ADHD+ODD, NONPROB) nested within person (level-1) with instructional group, family income, and maternal depressive symptoms as level-2 predictors. We began by testing the model with only the between subjects level-2 predictors, then added the within subjects factor as a fixed effect. Subsequent similar models tested whether the effects were similar for mothers' ratings of both inattentive symptoms and hyperactive-impulsive symptoms. Additional hierarchical linear models were conducted to test the influence of instructions, family income, and maternal depressive symptoms on the average frequency of responses in each of the coding categories assessing mothers' knowledge of ADHD. Finally, ratings of the instructional materials from mothers in the Instruction Group were examined descriptively.

\section{Results}

\section{Covariate and Moderator Group Differences}

We first examined differences across the two groups of mothers on the potential covariates of level of problems in the mother's own child, and familiarity with ADHD prior to participating in the study (see Table 1 for means and SDs). A $t$-test indicated no significant differences across the groups in the mothers' familiarity with ADHD. In terms of where they had gained their knowledge, most mothers indicated they had gained information through the media (67\%), through contact with someone with the disorder $(50 \%)$, or from medical/scientific readings (34\%). Chi-square tests indicated no significant differences in how mothers obtained 
information across the Instructions and No Instructions Groups. There were no differences across groups in the mothers' reports of their own child's level of problems on any of the SDQ subscales, nor on the total score.

The level of maternal depressive symptoms did not differ significantly across the Instructions and No Instructions Groups. However, despite random assignment to group, family income was significantly higher in mothers who received the instructions, $t(98)=2.27, p=.03$. This difference is accounted for in the hierarchical linear models that test for the effects of instructions and the moderators on the mothers' ratings. The average family income for mothers in the Instruction Group fell between the categories of $\$ 50,000$ - $\$ 74,999$ and $\$ 75,000$ - \$99,999 ranges, and the average income for mothers in the No Instructions Group fell between the $\$ 35,000$ - $\$ 49.999$ and $\$ 50,000-74,999$ ranges. Ten percent of mothers reported incomes below $\$ 19,999$ and another $14 \%$ reported incomes below $\$ 34,999$. For comparison, the low income cutoff for four-person households in large urban centers published by Statistics Canada (2010a) is approximately $\$ 26,000$, and the median family income in Vancouver in 2008 was $\$ 68,670$ (Statistics Canada, 2010b).

\section{Mothers' Ratings of Child Behavior}

As noted above, hierarchical linear modeling was used to analyze the data where the mothers' inaccuracy scores from the DVDs of the four types of child behavior were nested within person (level-1) with instructional group, family income, and maternal depressive symptoms as level-2 predictors. The intercept only model had an interclass correlation of .22. This indicates that $22 \%$ of the variance in behavior ratings was between subjects while $78 \%$ of the variance was within subjects. We began by testing the model with only the between subjects level-2 predictors, then added the within subjects factor also as a fixed effect. The between 
subjects predictors model (Model 1) included instructional group, family income, and maternal depressive symptoms as well as all two and three way interactions. Instructional group had no significant main effect on the inaccuracy scores, $\beta=-0.09$, ns. Family income had a significant negative effect, $\beta=-0.146, p<.05$, but was qualified by a significant interaction between instruction and income, $\beta=0.22, p<.05$. The main effect and all interactions with depressive symptoms were not significant. As depressive symptoms had no effect on the interaction between instructional group and family income and no relationship with the inaccuracy scores, it was only included as a covariate when testing the simple effects of income.

To probe the interaction of family income and instructions, plots are shown in Figure 1 for mothers divided into those with family incomes one standard deviation below and above the mean. Tests of the simple effects of instructional group at both the higher and lower income levels indicated a significant effect of instructions in reducing the discrepancy between mother and observer ratings of ADHD for mothers with incomes one standard deviation below the mean, $\beta=-.28, p=.02$. The effect of instructions for mothers one standard deviations above the mean income was not significant, $\beta=.15, p=.17$. Thus, as predicted the instructions reduced the discrepancy between mother and objective observer ratings, although only for mothers with relatively lower family incomes.

In Model 2, a type of child behavior factor was added to test the within subjects effects. Depressive symptoms were examined only as a main effect. The ADHD only DVDs were used as the reference condition for the dummy codes to test the effects of behavioral co-morbidity on the mothers' inaccuracy scores. Means and standard deviations for the two groups for each of the four types of child behavior are shown in Table 2. Model 2 was a significant improvement in model fit over Model 1 by the Likelihood Ratio test, $\chi^{2}(9)=242.46, p<.001$. There was a 
significant main effect of type of child behavior; compared to the ADHD only condition, DVDs portraying ADHD co-morbid with anxious (ADHD+ANX) and oppositional (ADHD+ODD) behaviors led to higher discrepancy scores (mothers identified more symptoms than the observers), $\beta=.19, p<.05$ and $\beta=.47, p<.001$ respectively. This result occurred despite the fact that the level of ADHD symptoms displayed was constant across the ADHD, ADHD+ODD, and ADHD+ANX portrayals. Thus, as predicted, comorbid oppositional behaviors increased mothers' ratings of child ADHD symptoms, and so did comorbid anxious behaviors. However, the lack of a significant interaction of type of child behavior and instruction group indicated that, contrary to prediction, the instructional materials did not reduce the influence of the comorbid behaviors on the discrepancies between mothers' and observers' ratings.

Family income revealed a significant interaction with type of child behavior. In the ADHD only condition, the mothers' discrepancy scores were significantly negatively related to family income, $\beta=-.27, p<.01$. Using the ADHD only condition as the baseline comparison, the relation between income and discrepancy scores in the ADHD+ODD condition was significantly different from that for the ADHD only condition, $\beta=.18, p<.05$, but the relation between income and discrepancy scores in the ADHD+ANX condition did not differ significantly from the baseline relation in the ADHD only condition. Examination of the bivariate correlations between family income and mothers' discrepancy scores indicated that this relation was only significant for the ADHD only condition, $r(99)=-.25, p=.01$, while the discrepancy scores in the other three conditions were not significantly correlated with family income, ADHD+ANX $r(99)=-.11, p=.29$; ADHD+ODD $r(99)=-.08, p=.41$, NOR $r(99)=$ $-.01, p=.90$. Finally, the interaction between instructional group and family income did not differ significantly across the types of child behavior type. 
We also ran hierarchical linear models for inaccuracy scores for inattentive symptoms and hyperactive-impulsive symptoms treated as separated dependent variables. In both cases, the pattern of significant results was identical to that for the overall scores. In sum, the effects of instructions, family income, and depressive symptoms were similar across types of symptoms.

\section{Mothers' Knowledge of ADHD}

The frequency of mothers' responses to the questions regarding the nature of ADHD and how to rate ADHD also were analyzed using hierarchical linear models with instructional group, family income, and maternal depressive symptoms and all two and three way interactions as predictors. Means and SDs are shown in Table 3. The responses of two mothers were not available due to a failure to administer the questions, one in each of the Instruction and No Instruction Groups. For the category of Correct Terms and Guidelines, the model indicated only a significant effect for instruction group, $\beta=.49, p=.02$. As predicted, mothers in the Instruction Group were more accurate than mothers in the No Instruction Group. The model for mothers' General Descriptions of ADHD revealed an interaction between instructions and depressive symptoms, $\beta=.42, p=.05$, but no other significant effects. Examination of the simple effects indicated that for mothers with depressive symptoms more than $1 \mathrm{SD}$ above the mean, there was no effect of the instructions on their descriptions, $\beta=.12, p=.69$, but for mothers with depressive symptoms less than 1 SD below the mean, the instructions significantly reduced the frequency of their general descriptions, $\beta=-.34, p=.008$. Thus, on this measure, the instructions had an impact only on the responses of mothers who were relatively low in depressive symptoms. The model predicting the average frequency of Inaccurate Statements revealed no significant effects.

\section{Evaluations of the Instructional Materials}


After they had rated the DVDs of child behavior, mothers in the Instruction Group were asked to rate the instructional materials in terms clarity, engagingness, and helpfulness. These ratings are shown in Table 3. Correlations between these ratings and family income and maternal depressive symptoms indicated no significant relations. Overall, mothers generally found the instructional materials clear, engaging, and helpful.

\section{Discussion}

Providing mothers with instructions regarding the meaning of items on ADHD questionnaires and how to make these ratings significantly increased the accuracy of mothers' ratings of both inattentive and hyperactive-impulsive ADHD symptoms, although only for mothers with lower family incomes. However, the instructions significantly increased all mothers' use of correct terms and appropriate guidelines in their open-ended answers assessing knowledge of ADHD. We also replicated the negative halo effect that comorbid oppositional behaviors have on mothers' ratings of ADHD symptoms, and extended this to show that comorbid anxious behaviors also increase mothers' ratings of ADHD symptoms. Unfortunately, the instructions did not reduce the negative halo effects due to comorbid behaviors.

The moderation of the influence of the instructions by family income suggests that, as predicted, these instructions are most useful to mothers who may be less likely or able to access health information or resources. Indeed, mothers from higher income families, even in the no instruction group, showed relatively better agreement with the objective observers (less discrepancy) suggesting less of a need for the instructional materials. Although further study is needed to illuminate which aspects of lower income translate into the greater advantage for the instructions, it is encouraging that the instructions were most effective for mothers who are most likely to be in need, both of information and services for ADHD. The only caveat to this 
conclusion is the fact that the instructions were equally effective across all levels of income when we examined the open-ended responses assessing knowledge of ADHD. Perhaps this more knowledge-based measure was less sensitive to the difficulties that mother from lower income families have in identifying ADHD behaviors. An additional comment is needed with regarding to the nature of the sample. Although the sample included a range of incomes and appeared representative of families in British Columbia, there were relatively few families at the very low levels of income. Thus, further testing which such groups is warranted, especially as low income families are likely to comprise a higher proportion of families in need of ADHD services.

Interestingly, contrary to our prediction, the mothers' level of depressive symptoms was not related to the discrepancy between mother and observer ratings. However, for mothers' openended responses regarding knowledge of ADHD, the instructions did interact with level of depressive symptoms. For mothers with lower levels of depressive symptoms, the instructions reduced their use of general descriptions, but for mothers who were more depressed the instructions had no effect on the number of general descriptions of ADHD provided. We speculate that mothers who were more depressed may have been providing more imprecise and vague answers regarding ADHD and that the instructions were not effective in reducing this tendency. In contrast, for mothers with lower levels of depression, although with no instructions they also gave relatively high levels of such general descriptions, with instructions these were replaced by more concise and precise answers. Although limited to only this one measure, this interaction indicates a potential limitation to the use of the instructions with mothers who are compromised by depressive symptoms.

An important finding from our study concerns the negative influence of comorbid child behaviors on mothers' ratings of ADHD symptoms, known as the negative halo effect. First, we 
replicated the findings from several previous studies showing that the presence of oppositional behaviors increases ratings of ADHD symptoms in the child (e.g., Abikoff et al., 1993; Hartung et al., 2006; 2010; Freeman, Johnston, \& Barth, 1997). We also demonstrated that the presence of anxious behaviors has a similar effect. Mothers rated more ADHD symptoms in children who were shown as also having anxious behaviors, compared to children showing only ADHD symptoms. Thus, the presence of anxious behaviors does not seem to offer an alternate explanation for ADHD symptoms and does not work to reduce the likelihood of these symptoms being endorsed. On the contrary, it appears that anxious behaviors may be mistaken for ADHD symptoms (e.g., nervousness being mistaken as restlessness) or there may be a general negative halo effect in operation, such that the mere presence of any additional problem behaviors increases ratings of ADHD symptoms. Separation of these two possibilities awaits further study. Regardless, our results suggest that the presence of comorbid anxious behaviors in children being assessed for ADHD should be considered as a possible biasing influence on parents' reports of their child's behavior. It should be noted that the conditions with comorbid child behaviors displayed, not only qualitatively different comorbid behaviors along with the ADHD behaviors, but also quantitatively more problem behaviors. Further testing is also needed to rule out whether quantity of problems, rather than their comorbid nature, may account for the findings.

The interaction of family income with type of child behavior suggests that although mothers with lower socioeconomic levels were more likely to identify more ADHD symptoms when the child was showing only ADHD groups, this relation diminished when the children were either not displaying any problems or when the comorbid problems were present. It is likely that the clearly nonproblematic nature of the NOR portrayals prevented any demonstration of bias in ratings, while the presence of the comorbidities appeared to over-ride the relation 
between family income and more negative perceptions of children such that all mothers endorsed more ADHD symptoms in these comorbid conditions.

\section{Limitations}

The non-referred nature of the sample of mothers in this study prevents us from speaking with confidence regarding whether the instructions would have similar effects among mothers whose children were referred for ADHD assessment. For example, the low rate of inaccurate statements about ADHD provided in the open-ended responses suggests that we might find even more robust effects of these instructional materials among parents who know less about the disorder or who hold inaccurate beliefs about the disorder. Obvious next steps are to continue to evaluate the instructional materials in such groups, as well as with fathers and other assessors, such as teachers. We are also limited in being able to draw conclusion restricted to boys. Although experts rated the instructional materials as appropriate for use with both genders, explicit testing of the effectiveness in samples of girls is clearly needed. In addition, the mothers in this study rated standard child behavior stimuli, rather than their own children's behavior. While this methodology has clear advantages in terms of holding constant the child behavior being rated, it presents another obstacle to assessing the generalizability of the findings to the clinical context where objective criteria are less likely to be available. The instructional materials must now be evaluated under "real world" conditions among parents who are asked to evaluate their own children during the course of an ADHD assessment and where clinicians must resolve discrepancies across reports without the benefit of any clear, objective standard. We have recently completed a study evaluating these instructions in sample of clinic-referred families and the preliminary results confirm the effects of the instructions in improving the agreement 
between mothers' and others' ratings of ADHD behaviors in the mothers' own child (Johnston, Weiss, Murray, \& Miller, 2010), however, further analysis and replication is definitely needed. The results of this study also illustrate areas in which further efforts are needed to enhance the accuracy of parental reports of child ADHD symptoms. Although the instructions were useful, they were not effective in combating the biasing influence of comorbid child problems on maternal ratings. Results from the open-ended responses also suggested that, at least on some dimensions, mothers with higher levels of depressive symptoms were less influenced by the instructions. Although information regarding how parents should distinguish ADHD symptoms from behaviors reflective of other disorders is included in the current version of the instructions, it is obviously not sufficient. Further elaboration of this material (e.g., providing additional examples of comorbid behaviors that should not be mistaken for ADHD), or alternate ways of conveying this information (e.g., explicitly instructing parents to focus on ADHD behaviors and to ignore co-occurring behaviors) may be useful. Given that the interaction of maternal depressive symptoms and instructions appeared on only one measure, we are reluctant to draw firm conclusions regarding the inappropriateness of the instructions for mothers with higher levels of depression. Instead, further testing of this effect, particularly within a clinical sample where levels of maternal depression may be much higher is needed.

Finally, the study is limited by its reliance on family income as the indicator of family socioeconomic status. Current consensus is that socioeconomic status is a multidimensional construct, reflecting a variety of factors including income, but also education, occupation, and resources (Braveman et al., 2005; Callahan \& Eyberg, 2010). Which aspect of socioeconomic status, perhaps health literacy or access to mental health services, is most important in relation to the instructional effects remains to be explored. In addition, there emerging evidence that 
socioeconomic status is contextually-bound (Braveman et al., 2005), and this points to the need to test the influence of these instructions and their interaction with multiple indicators of socioeconomic status in samples of families from different geographic regions, living in rural and urban settings, and of multiple ethnicities.

\section{Clinical Implications}

If further development and testing of these instructional materials continues to support their effectiveness in improving the accuracy of parents' ratings, they will offer a useful tool to enhance the validity of parental reports as used in the assessment of ADHD. This could be implemented in several different ways. Clinicians using ADHD rating scales with parents might add the pamphlet and/or audio/visual presentation to improve the accuracy of parents' reports. We believe the instructional materials are practical and efficacious strategy for several reasons. Interviews or teaching around rating scale interpretation is costly, and the rating scales used to assess ADHD are already widely used and well-established (e.g., DuPaul et al., 1998; Gadow \& Sprafkin, 2002; Pelham et al., 1987). Instructional materials could easily be incorporated into clinical practice and used in conjunction with any of these rating scales or interviews to enhance their value. In addition, the mothers' evaluations of the instructional materials are encouraging indicators that the materials are likely to be well received by parents. We believe the use of such instructions may prove particularly critical in the early stages of ADHD assessment when parents, particularly those from lower socioeconomic groups, may have relatively little accurate knowledge about the disorder. The instructional materials also are likely to be useful in situations where clinicians are making categorical diagnosis where differences of one or two items can be interpreted as indicating that ADHD is "present" or "absent," and when inaccuracy in parent ratings may be based on misunderstanding of the items. 


\section{References}

Abikoff, H., Courtney, M., Pelham, W. E., Jr., \& Koplewicz, H. S. (1993). Teachers' ratings of disruptive behaviors: The influence of halo effects. Journal of Abnormal Child Psychology, 21, 519-533.

American Psychiatric Association. (2000). Diagnostic and Statistical Manual of Mental Disorders (4th ed. Text Revision). Washington, DC: Author.

Angold, A., Costello, E. J., \& Erkanli, A. (1999). Comorbidity. Journal of Child Psychology and Psychiatry, 40, 57-87.

Barkley, R. A. (2006). Attention-deficit hyperactivity disorder: A handbook for diagnosis and treatment (3rd ed.). New York: Guilford Press.

Bauermeister, J. J., Shrout, P. E., Ramirez, R., Bravo, M., Alegria, M., Martinez-Taboas, A., et al. (2007). ADHD correlates, comorbidity, and impairment in community and treated samples of children and adolescents. Journal of Abnormal Child Psychology, 35, 883898.

Burns, G. L., Walsh, J. A., Gomez, R., \& de Moursa, M. A. (2003). Understanding source effects in ADHD Rating Scales: Reply to DuPaul (2003). Psychological Assessment 15, 118 119.

Bussing, R., Gary, F. A., Mills, T. L., \& Wilson Garvan, C. (2007). Cultural variations in parental health beliefs, knowledge, and information sources related to attentiondeficit/hyperactivity disorder. Journal of Family Issues, 28, 291-318.

Bussing, R., Fernandez, M., Harwood, M., Hou, W., Garvan, C. W., Eyberg, S. M., \& Swanson, J. M. (2008). Parent and teacher SNAP-IV ratings of attention deficit hyperactivity 
disorder symptoms: Psychometric properties and normative ratings from a school district sample. Assessment, 15, 317-328.

Braveman, P. A., Cubbin, C., Egerter, S., Chideya, S., Marchi, K. S., Metzler, M., \& Posner, S. (2005). Socioeconomic status in health research: One size does not fit all. Journal of the American Medical Association, 294, 2879-2888.

Callahan, C. L., \& Eyberg, S. M. (2010). Relations between parenting behavior and SES in a clinical sample: Validity of SES measures. Child and Family Behavior Therapy, 32, 125138.

Chi, T. C., \& Hinshaw, S. P. (2002). Mother-child relationships of children with ADHD: The role of maternal depressive symptoms and depression-related distortions. Journal of Abnormal Child Psychology, 30, 387-400.

Collett, B. R., Ohan, J. L., \& Myers, K. M. (2003). Ten-year review of rating scales: V. Scales assessing Attention-Deficit/Hyperactivity Disorder. Journal of the American Academy of Child and Adolescent Psychiatry, 42, 1015-1037.

Conners, C. K., Sitarenios, G., Parker, J. D. A., \& Epstein, J. N. (1998), The revised Conners' Parent Rating Scale (CPRS-R): Factor structure, reliability, and criterion validity. Journal of Abnormal Child Psychology, 26, 257-268.

Derogatis, L. R. (1993). Brief Symptom Inventory: Administration, Scoring, and Procedures Manual. Minneapolis: National Computer Systems, Inc.

DuPaul, G. J., Power, T. J., Anastopoulos, A. D., \& Reid, R. (1998). ADHD Rating Scale-IV: Checklists, norms, and clinical interpretation. New York: Guilford Press. 
Freeman, W. S., Johnston, C., \& Barth, F. M. (1997). Parent attributions for inattentiveoveractive, oppositional-defiant, and prosocial behaviours in children with attention deficit hyperactivity disorder. Canadian Journal of Behavioural Science, 29, 239-248.

Gadow, K. D., Loney, J., Sprafkin, J., Salisbury, H., Azizian, A. \& Schwartz, J. (2004). Comparison of ADHD symptom subtypes as source-specific syndromes. Journal of Child Psychology and Psychiatry, 45, 1135-1149.

Gadow, K. D., \& Sprafkin, J. (2002). Child Symptom Inventory 4: Screening and Norms Manual. Stony Brook, NY: Checkmate Plus.

Gomez, R., Burns, L. G., Walsh, J. A., \& De Moura, M. A. (2003). Multitrait-multisource confirmatory factor analytic approach to the construct validity of ADHD rating scales. Psychological Assessment, 15, 3-16.

Goodman, R. (1997). The Strengths and Difficulties Questionnaire: A research note. Journal of Child Psychology and Psychiatry, 38, 581-586.

Hartung, C. M., Lefler, E. K., Tempel, A. B., Armendariz, M. L., Sigel, B. A., \& Little, C. S. (2010). Halo effects in ratings of ADHD and ODD: Identification of susceptible symptoms. Journal of Psychopathology and Behavioral Assessment, 32, 128-137.

Hartung, C. M., Van Pelt, J. C., Armendariz, M. L., \& Knight, L. A. (2006). Biases in ratings of disruptive behavior in children: effects of sex and negative bias. Journal of Attention Disorders, 9, 620-630.

Jackson, D. A., \& King, A. R. (2004). Gender differences in the effects of oppositional behavior on teacher ratings of ADHD symptoms. Journal of Abnormal Child Psychology, 32, 215224.

Johnston, C., \& Short, K. H. (1993). Depressive symptoms and perceptions of child behavior. 
Journal of Clinical and Social Psychology, 12, 164-181.

Johnston, C., Weiss, M., Murray, C., \& Miller, N. (June, 2011). Rating instructions improve agreement between mother and other reports of child ADHD. Poster presented to the International Society for Research in Child and Adolescent Psychopathology.

Langberg, J. M., Epstein, J. N., Simon, J. O., Loren, R. E. A., Arnold, L. E., Hechtman, L., et al. (2010). Parent agreement on ratings of children's Attention Deficit/Hyperactivity Disorder and broadband externalizing behaviors. Journal of Emotional and Behavioral Disorders, 18, 41-50.

Larson, K., Russ, S. A., Kahn, R. S., \& Halfon, N. (2011). Paterns of comorbidity, functioning, and service use for US children with ADHD, 2007. Pediatrics, 127, 462-470.

Lee, C. M. (2006). Involving fathers in the delivery of psychological services. Cognitive and Behavioral Practice, 13, 40-41.

McConaughy, S. H., \& Achenbach, T. M. (2009). Guide for the ASEBA Direct Observation Form. Burlington, VT: University of Vermont, Research Center for Children, Youth, \& Families.

Pelham, W. E., Jr., Fabiano, G. A., \& Massetti, G. M. (2005). Evidence-based assessment of Attention Deficit Hyperactivity Disorder in dhildren and adolescents. Journal of Clinical Child and Adolescent Psychology, 34, 449-476.

Pelham, W. E., Jr., Gnagy, E. M., Greenslade, K. E., \& Milich, R. (1992). Teacher ratings of DSM-III-R symptoms for the disruptive behavior disorders. Journal of the American Academy of Child and Adolescent Psychiatry, 31, 210-218.

Power, T. J., Doherty, B. J., Panichelli-Mindel, S. M., Karustic, J. L., Eirabldi, R. B., Anastopoulos, A. D., \& DuPaul, G. J. (1998). The predictive validity of parent and 
teacher reports of ADHD symptoms. Journal of Psychopathology and Behavioral Assessment, 20, 57-81.

Rieppi, R., Greenhill, L. L., Ford, R. E., Chuang, S., Wu, M., Davies, M., et al. (2002). Socioeconomic status as a moderator of ADHD treatment outcomes. Journal of the American Academy of Child \& Adolescent Psychiatry, 41, 269-277.

Solanto, M. V., \& Alvir, J. (2009). Reliability of DSM-IV symptom ratings of ADHD: Implications for DSM-V. Journal of Attention Disorders, 13, 107-116.

Statistics Canada. (2010a). Income Research Paper Series: Low Income Lines, 2008-2009. (Catalogue number 75F0002M - No. 005). Retrieved from Statistics Canada website: http://www.statcan.gc.ca/pub/75f0002m/75f0002m2010005-eng.pdf

Statistics Canada (2010b). Median total income, by family type, by census metropolitan area. CANSIM table 111-0009. Retrieved from Statistics Canada website: http://www40.statcan.ca/101/cst01/famil107a-eng.htm

Wolraich, J. L., Lambert, E. W., Bickman, L., Simmons, T., Doffing, M. A., \& Worley, K. A. (2004). Assessing the impact of parent and teacher agreement on diagnosisng Attention Deficit Hyperactivity Disorder. Journal of Developmental and Behavioral Pediatrics, 25, 41-47. 
Table 1 Descriptive information

Variable $\quad$ No Instructions $(n=51) \quad$ Instructions $(n=49)$

Mothers' ethnicity - frequency (percentage)

$\begin{array}{lcc}\text { European/N. American } & 27(53 \%) & 28(57 \%) \\ \text { Asian } & 15(29 \%) & 14(29 \%) \\ \text { Other } & 9(18 \%) & 7(14 \%)\end{array}$

\begin{tabular}{|c|c|c|}
\hline & $\mathrm{M}(\mathrm{SD})$ & $\mathrm{M}(\mathrm{SD})$ \\
\hline Child age in months & $101.37(24.30)$ & $111.02(25.60)$ \\
\hline Mother age in years & $38.68(6.13)$ & $40.59(5.78)$ \\
\hline Familiar with ADHD ${ }^{b}$ & $2.14(.60)$ & $2.29(.65)$ \\
\hline \multicolumn{3}{|l|}{ Own child's problems ${ }^{a}$} \\
\hline Emotional & $.43(.44)$ & $.40(.33)$ \\
\hline Conduct & $.27(.29)$ & $.30(.33)$ \\
\hline Hyperactivity & $.59(.44)$ & $.57(.51)$ \\
\hline Peers & $.37(.42)$ & $.39(.35)$ \\
\hline Total & $.41(.27)$ & $.41(.29)$ \\
\hline Mother depressive symptoms ${ }^{\mathrm{d}}$ & $.69(.79)$ & $.47(.51)$ \\
\hline Family Income ${ }^{c}$ & $4.53(1.79)$ & $5.31(1.62)$ \\
\hline
\end{tabular}

a Mothers' familiarity with ADHD prior to the study, rated on a 1 to 4 score; ADD ${ }^{\mathrm{d}}$ Average item rating on the Depression subscale of the Brief Symptom Inventory;

${ }^{\mathrm{e}}$ Average item rating on the Strengths and Difficulties Questionnaire. 
Table 2

Inaccuracy Scores for Ratings of Child ADHD Symptoms and Descriptions of ADHD

Child Behavior

No Instructions

Instructions

Stimuli

$\underline{\text { Inaccuracy Scores }}$

NOR

$-.06(.18)$

$-.05(.18)$

ADHD

$.61(.64)$

$.43(.62)$

ADHD+ANX

.78 (.69)

$.68(.65)$

ADHD+ODD

$1.05(.73)$

$.89(.60)$

Descriptions of ADHD

Correct Terms

$.42(.78)$

$.98(1.16)$

Descriptions

$6.28(3.54)$

4.77 (3.14)

Inaccurate Stmts

$.18(.57)$

$.27(.89)$

Note: Inaccuracy Scores are the average difference between the mothers' and observers' ratings of the two children portraying each type of child behavior. $\mathrm{NOR}=$ normal behavior; $\mathrm{ADHD}=$ Attention-Deficit/Hyperactivity Disorder symptoms; ADHD+ANX = Attention-

Deficit/Hyperactivity Disorder symptoms and anxious behaviors; ADHD+ODD = Attention-

Deficit/Hyperactivity Disorder symptoms and oppositional behaviors. 
Table 3

Mothers' Ratings of the Instructional Materials

Variable

Written Pamphlet

Understandable

$6.67(.55)$

Engaging

$6.04(1.06)$

Helpful

$6.22(.82)$

Slide Presentation

Understandable

$6.18(1.03)$

Engaging

$5.96(1.20)$

Helpful

$5.86(1.51)$

Note: Ratings on a 1 (low) to 7 (high) scale. 
Figure 1

Inaccuracy Scores By Instructional Group and Family Income

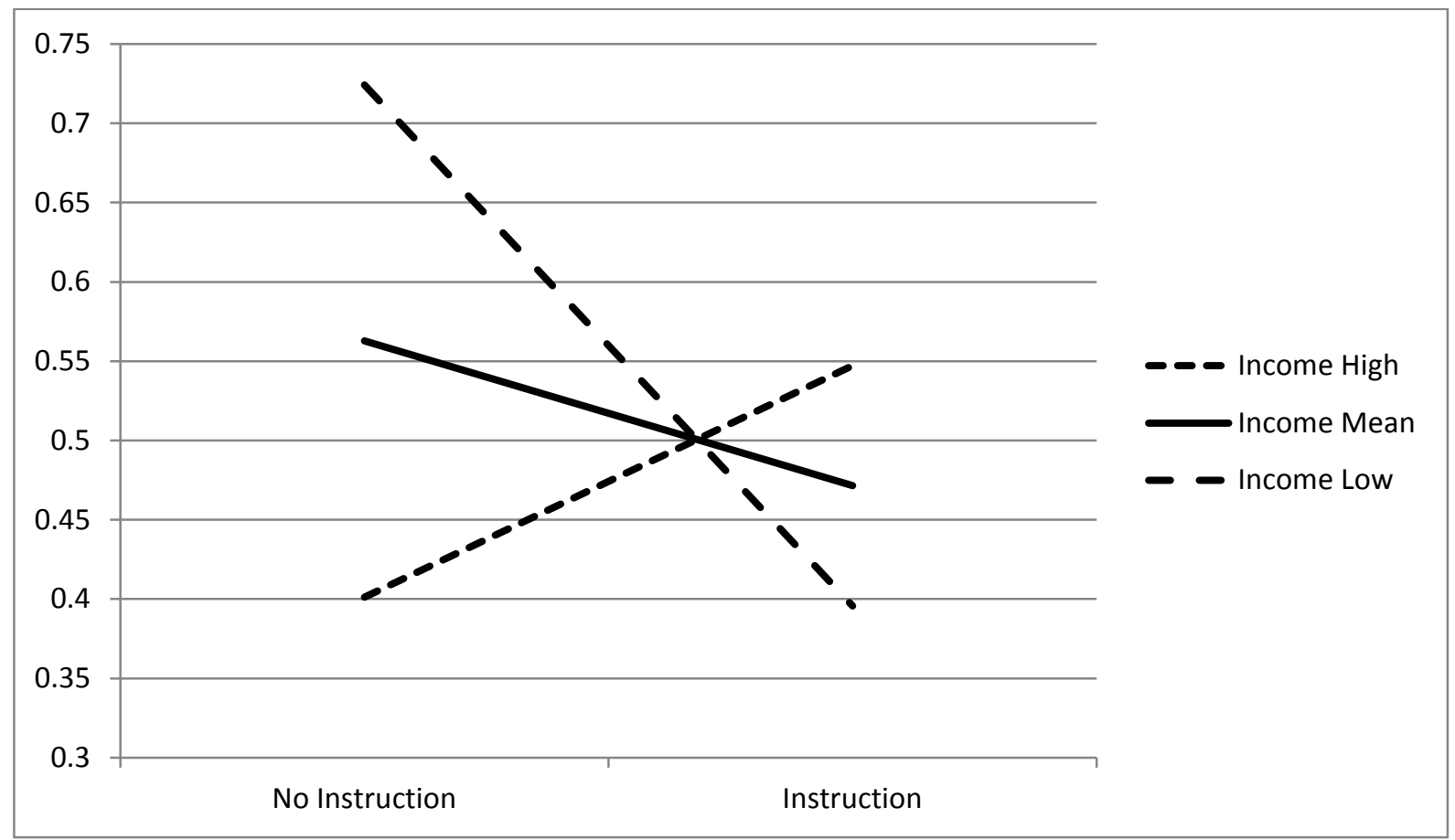


Figure 2

Frequency of Descriptions of ADHD By Instructional Group and Maternal Depressive Symptoms

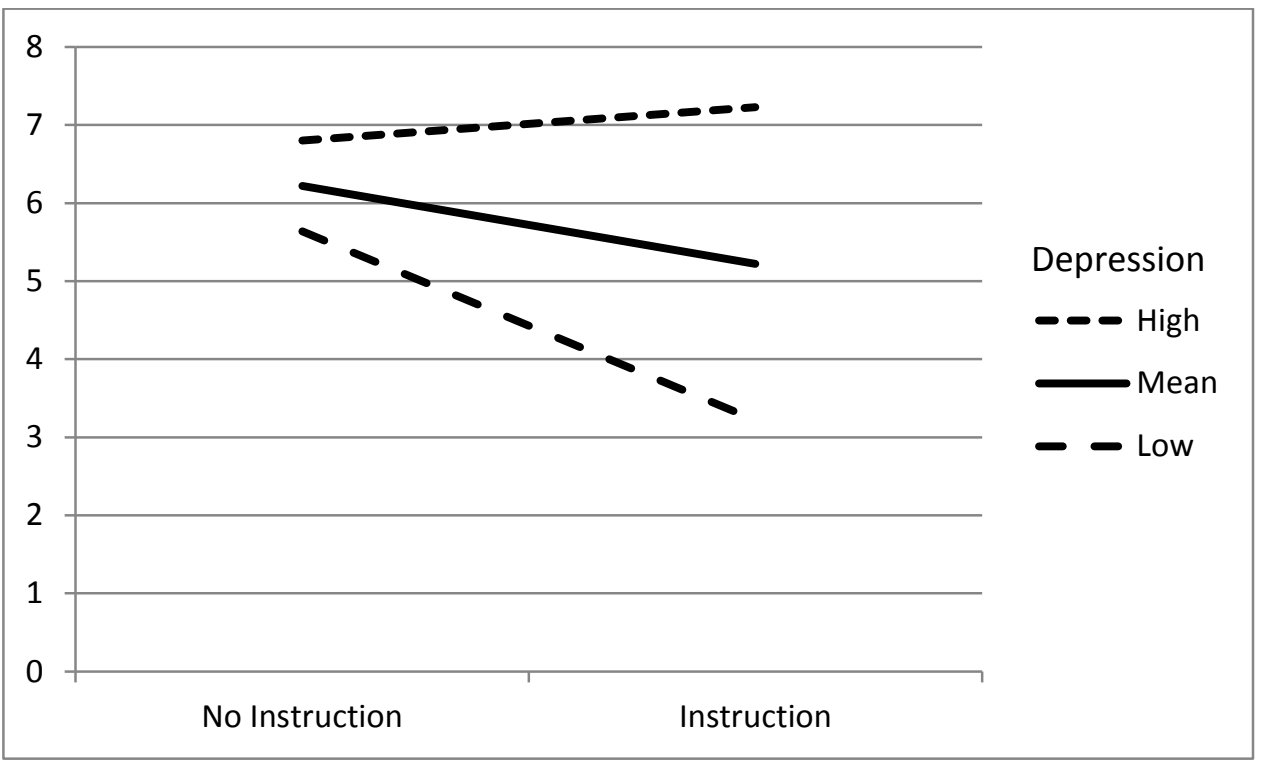

